\title{
Guided Relinking of Graph Pyramids *
}

\author{
R. Glantz and W.G. Kropatsch \\ Institute for Computer Aided Automation \\ Pattern Recognition and Image Processing Group \\ Vienna University of Technology \\ Favoritenstr. 9, 183/2, A-1040 Vienna, Austria \\ $\{$ glz,krw\}@prip.tuwien.ac.at
}

\begin{abstract}
In this paper we propose a new method to relink graph pyramids by local relinking operations in an iterated parallel way. By representing graph pyramids as bases of valuated matroids, the goal of the relinking is expressed by a valuation on the corresponding matroid. This valuation guides the local relinking operations. The valuation attains its global maximum if none of the local relinking operations yields higher values. The new method is used for an adaption of graph pyramids towards having a given receptive field.
\end{abstract}

\section{Introduction}

To perceive an image is to transform it [Ser82]. In order to allow a clear distinction between transformations of image structure and transformations of image contents, we first represent the image as an attributed graph forming the base level of a graph pyramid. A common way to construct the base level graph is to create a vertex for each pixel and to let the edges represent the 4-connectivity of the pixel array. The attributes of the vertices, edges and faces are derived from the gray values or colors of the pixels. The other levels of the pyramid are formed by subsequent dual graph contractions Kro95a controlled by application defined models. A local function, the so called reduction function, derives the attributes of the current level from the level below. In all levels the attributes represent the image contents, while the structure of the image is given by the graph without the attributes. The graphs on the higher levels of the pyramid yield more and more abstract descriptions of the underlying image. However, the construction of the graph pyramid should not be restricted to a bottom-up procedure. The alternatives as given by a model usually induce constraints on neighborhoods in the graph pyramid. Holding to the separation of structure and contents we extend the influence of the model by allowing

1. relinking of the pyramid without adjusting the contents,

2. contents adjustments and classification without relinking.

\footnotetext{
* This work has been supported by the Austrian Science Fund (FWF) under grant S7002-MAT.
} 
These transformations are also utilized to increase the robustness of the pyramid. This paper is devoted to efficiently perform the relinking by iterated parallel transformations (IPT) [Sch97. A variable linking of regular pyramids was first described in [BHR81]. An extension to irregular hierarchies of graphs is shown in Nac95. IPT for contents adjustment and classification, i.e. relaxation, has been applied to hierarchies of graphs in [WH96]. Since dual graph contraction is an IPT towards abstraction, the IPT considered so far can be organized in the triangle depicted in Fig. 1.

The paper is organized as follows. Section 2 is devoted to the construction of graph pyramids by dual graph contraction. In Section 3 we arrive at a definition of local relinking operations on graph pyramids. The definition is based on the representation of graph pyramids as bases of matroids. Section 4 introduces valuations on matroids. The valuations are utilized to guide the local relinking operations. In Section 5 we apply the relinking to the adaption of graph pyramids towards having a given receptive field. We conclude in Section 6 .

\section{Dual Graph Contraction}

The construction of graph pyramids by dual graph contraction (see Fig. 2) is described in Kro95a]. Let $G_{0}=\left(V_{0}, E_{0}\right)$ and $\overline{G_{0}}=\left(\overline{V_{0}}, \overline{E_{0}}\right)$ denote a pair of plane graphs, where $\overline{G_{0}}$ is the dual of $G_{0}$. Dual graph contraction consists of two steps: dual edge contraction and dual face contraction. Dual edge contraction is specified by a subset $F_{0}$ of $E_{0}$, such that the edges of $F_{0}$ form a spanning forest of $G_{0}$. The trees of the spanning forest are referred to as contraction kernels. In Fig. $2 \mathrm{a}$ the non-trivial contraction kernels are emphasized. Each contraction kernel $T_{0}$ of $F_{0}$ is contracted to one vertex $v_{1}$ of the graph $G_{1}=\left(V_{1}, E_{1}\right)$ on the next level of the graph pyramid. For each vertex $v_{0}$ of $T_{0}$ the vertex $v_{1}$ is called parent of $v_{0}$ and $v_{0}$ is called the child of $v_{1}$. Each edge of $E_{1}$ corresponds to exactly one edge in $E_{0}$, which does not belong to a contraction kernel. Let $\overline{F_{0}}$

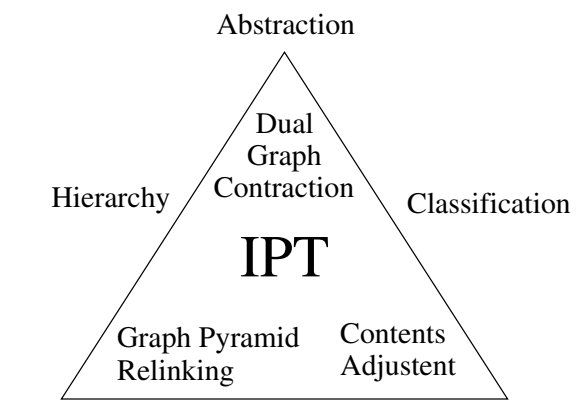

Structure

Contents

Fig. 1. Iterative parallel transformations on graph pyramids. 


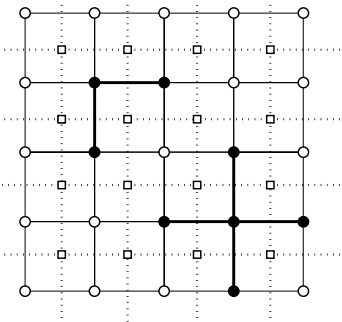

(a) $\left(G_{0}, \bar{G}_{0}\right)$

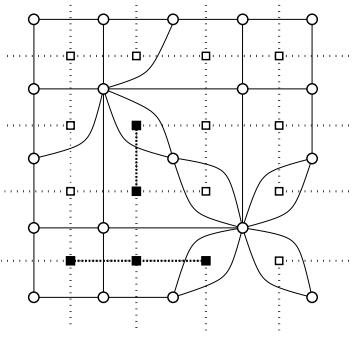

(b) $\left(G_{1}, \bar{G}_{1}\right)$

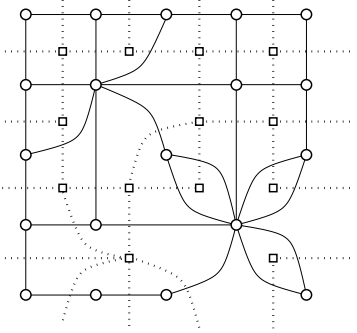

(c) $\left(G_{2}, \bar{G}_{2}\right)$

Fig. 2. Dual graph contraction.

denote the set of edges in $\bar{E}_{0}$, which are dual to the edges in $F_{0}$. Set $\overline{E_{1}}:=\overline{E_{0}} \backslash \overline{F_{0}}$ and $\overline{G_{1}}:=\left(\overline{V_{0}}, \overline{E_{1}}\right)$. Note that $G_{1}$ and $\overline{G_{1}}$ form a dual pair of plane graphs.

The second step, called dual face contraction, is specified by a subset $\overline{F_{1}}$ of $\overline{E_{1}}$. The edges of $\overline{F_{1}}$ are required to form a spanning tree of $\overline{G_{1}}$. In Fig. $2 \mathrm{~b}$ the edges of $\overline{F_{1}}$ are emphasized. Analogous to dual edge contraction, we generate $\overline{G_{2}}$ and set $G_{2}:=\left(V_{1}, E_{2}\right)$ with $E_{2}:=E_{1} \backslash F_{1}$. Each vertex in $G_{2}$ has exactly one child in $G_{1}$, i.e. the vertex itself. The graphs $G_{2}$ and $\overline{G_{2}}$ form another dual pair of plane graphs. In [Kro95a] the role of dual face contraction is confined to the removal of faces bounded by less than three edges. In the following we will drop this restriction in order to apply the theory of matroids in a general way. Subsequent parallel edge [face] contraction steps may be summarized by a single edge [face] contraction step.

Each vertex in the graph pyramid represents a connected set of base level vertices, the so called receptive field. The receptive field of a base level vertex contains exactly the vertex itself. For each vertex $v_{k}$ on the level $k \geq 1$ the receptive field $R F\left(v_{k}\right)$ is defined by all vertices in the base level of the pyramid which lead to $v_{k}$ by climbing the pyramid from children to parents. In Fig. 3 the odd levels are omitted.

$R F\left(v_{0}\right)=\left\{v_{0}\right\}$ for $v_{0} \in V_{0}$, $R F\left(v_{k}\right)=\bigcup\left(R F\left(v_{k-1}\right) \mid v_{k-1}\right.$ is child of $\left.v_{k}\right), \quad k>0$.

Note that the receptive fields in the graph pyramid do not overlap, since all vertices (except the apex) have exactly one parent.

\section{Representation of Graph Pyramids as Bases of Matroids}

Let $G_{0}$ and $\bar{G}_{0}$ denote a pair of plane graphs and assume $\mathcal{P}=\left(G_{0}, G_{1}, \ldots, G_{2 n}\right)$ and $\overline{\mathcal{P}}=\left(\bar{G}_{0}, \bar{G}_{1}, \ldots, \bar{G}_{2 n}\right)$ to be graph pyramids constructed on top of the pair 


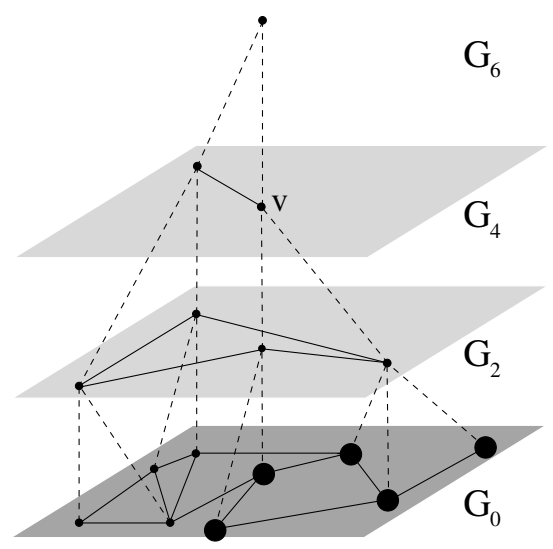

Fig. 3. The vertices forming the receptive field of $v$ are enlarged.

$\left(G_{0}, \bar{G}_{0}\right)$ by dual graph contractions. We also assume that the apex $G_{2 n}$ is a graph with one vertex and zero edges. Let $G_{i}=\left(V_{i}, E_{i}\right)$ for all $0 \leq i \leq 2 n$. The edge set $E_{0}$ is required to be non-empty. The domain of all graph pyramids with the above properties is denoted by $\mathcal{D}\left(G_{0}, 2 n\right)$. For each edge $e \in E_{0}$ let $l(e)$ denote the maximal level of $\mathcal{P}$ which contains $e$, i.e.

$$
l(e):=\max \left\{l \mid e \in E_{l} \backslash E_{l+1}\right\} .
$$

The construction of the graph pyramid is determined by the above assignment of labels from $L:=\{0,1, \ldots, 2 n-1\}$ to the edges in $E_{0}$ (similar to Kro95b). The assignments are expressed by subsets of $E_{0} \times L$. Let $B$ denote a subset of $E_{0} \times L$. We set

$$
E^{0}(B):=\left\{e \in E_{0} \mid \exists j \text { with }(e, j) \in B \text { and } j \equiv 0 \bmod 2\right\} .
$$

If $B=\left\{(e, l(e)) \mid e \in E_{0}\right\}$, where $l(\cdot)$ refers to the construction of a graph pyramid, then

- $\forall e \in E_{0}$ exists exactly one $l$ with $(e, l) \in B$ and

- $E^{0}(B)$ forms a spanning tree in $E_{0}$.

Conversely, let $B \subset E_{0} \times L$. If $B$ fulfills the above two items, then $B$ defines a labeling of edges from $E_{0}$, which describes the construction of a graph pyramid. This follows from the fact, that $E^{0}(B)$ forms a spanning tree in $E_{0}$ if and only if

$$
E^{1}(B):=\left\{e \in E_{0} \mid \exists j \text { with }(e, j) \in B \text { and } j \equiv 1 \bmod 2\right\}
$$

forms a maximal edge set in $G_{0}$, which is not a cutset TS92] (a cutset of a connected graph $G$ is a minimal set of edges of $G$ such that its removal from $G$ disconnects $G$ ). Hence, the edges in $\overline{G_{0}}$, which are dual to $E^{1}(B)$ form a spanning tree $\overline{E^{1}(B)}$ in $\overline{G_{0}}$. In conjunction with the labels from $B$, the spanning trees 
$E^{0}(B)$ and $\overline{E^{1}(B)}$ define the contraction kernels for the dual edge contraction and the dual face contraction respectively.

Let $\mathcal{B}$ denote the collection of all subsets of $E_{0} \times L$ which describe constructions of graph pyramids in $\mathcal{D}\left(G_{0}, 2 n\right)$. Note that $\mathcal{B} \neq \emptyset$ because of $E_{0} \neq \emptyset$. The following theorem states an exchange property for sets in $\mathcal{B}$.

Theorem 1. Let $B, B^{\prime} \in \mathcal{B}$. For each $b \in B \backslash B^{\prime}$ there exists $b^{\prime} \in B^{\prime} \backslash B$ such that $B \backslash\{b\} \cup\left\{b^{\prime}\right\} \in \mathcal{B}$.

Proof: It suffices to show that $E^{0}\left(B \backslash\{b\} \cup\left\{b^{\prime}\right\}\right)$ forms a spanning tree of $G_{0}$ or that $E^{1}\left(B \backslash\{b\} \cup\left\{b^{\prime}\right\}\right)$ forms a maximal non-cutset of $G_{0}$.

- Case $b=(e, l)$ with $l>0$ : In the fundamental circuit [TS92] of $E^{0}\left(B^{\prime}\right) \cup\{e\}$ there exists $e^{\prime} \notin E^{0}(B)$ (since $E^{0}(B)$ contains no cycles). Let $l^{\prime} \in L$ denote the unique number with $\left(e^{\prime}, l^{\prime}\right) \in B^{\prime}$ and set $b^{\prime}:=\left(e^{\prime}, l^{\prime}\right)$. Since $e^{\prime} \notin E^{0}(B)$, it follows that $e^{\prime} \neq e$. This implies $b^{\prime} \neq b$ and (because of $\left.e^{\prime} \in E^{0}\left(B^{\prime}\right) \cup\{e\}\right) e^{\prime} \in E^{0}\left(B^{\prime}\right)$, i.e. $l^{\prime}>0$. Since $e$ and $e^{\prime}$ belong to the same cycle of $E^{0}\left(B^{\prime}\right) \cup\{e\}$ and have positive labels, it follows that $E^{0}\left(B \backslash\{b\} \cup\left\{b^{\prime}\right\}\right)$ forms a spanning tree of $E_{0}$.

- Case $b=(e, l)$ with $l<0$ : The set $E^{1}\left(B^{\prime}\right) \cup\{e\}$ forms a cutset of $G_{0}$. Since $E^{1}(B)$ contains no cutset, there exists $e^{\prime} \in E^{1}\left(B^{\prime}\right) \cup\{e\}, e^{\prime} \notin E^{1}(B)$. Let $l^{\prime} \in L$ denote the unique number with $\left(e^{\prime}, l^{\prime}\right) \in B^{\prime}$ and set $b^{\prime}:=\left(e^{\prime}, l^{\prime}\right)$. Since $e^{\prime} \notin$ $E^{1}(B)$, it follows that $e^{\prime} \neq e$. This implies $b^{\prime} \neq b$ and (because of $e^{\prime} \in E^{1}\left(B^{\prime}\right) \cup$ $\{e\}) e^{\prime} \in E^{1}\left(B^{\prime}\right)$, i.e. $l^{\prime}<0$. Since $e$ and $e^{\prime}$ belong to the cutset $E^{1}(B) \cup\left\{e^{\prime}\right\}$, it follows that $E^{1}\left(B \backslash\{b\} \cup\left\{b^{\prime}\right\}\right)$ is a maximal non-cutset.

Definition 1. For $B \in \mathcal{B}, b \in B, b^{\prime} \notin B$ the mapping modif $\left(B, b, b^{\prime}\right):=B \backslash$ $\{b\} \cup\left\{b^{\prime}\right\}$ is called local modification of $B$, if modif $\left(B, b, b^{\prime}\right) \in \mathcal{B}$.

The sets in $\mathcal{B}$, the so called bases, determine a matroid $\mathcal{M}:=\left(E_{0} \times L, \mathcal{I}\right)$ on $E_{0} \times L$, where

$$
\mathcal{I}:=\{I \subset B \mid B \in \mathcal{B}\}
$$

Ox192. Thus we may write $\mathcal{M}=\mathcal{M}(\mathcal{B})$. In Bru69 the exchange property of Theorem 1 is extended:

Theorem 2. Let $\mathcal{B}$ denote the collection of bases of a matroid and let $B, B^{\prime} \in \mathcal{B}$. For each $b \in B \backslash B^{\prime}$ there exists $b^{\prime} \in B^{\prime} \backslash B$ such that

- $B \backslash\{b\} \cup\left\{b^{\prime}\right\} \in \mathcal{B}$ and

- $B^{\prime} \backslash\left\{b^{\prime}\right\} \cup\{b\} \in \mathcal{B}$.

Theorem 2 implies that any $B \in \mathcal{B}$ can be adapted to any other $B^{\prime} \in \mathcal{B}$ by local modifications only.

If the construction of $\mathcal{P}$ is determined by $B$, each local modification of $B$ induces an operation on $\mathcal{P}$. We define:

Definition 2. An operation on a graph pyramid $\mathcal{P}$ is called local relinking operation, if it is induced by a local modification on a matroid base that describes the construction of $\mathcal{P}$. 


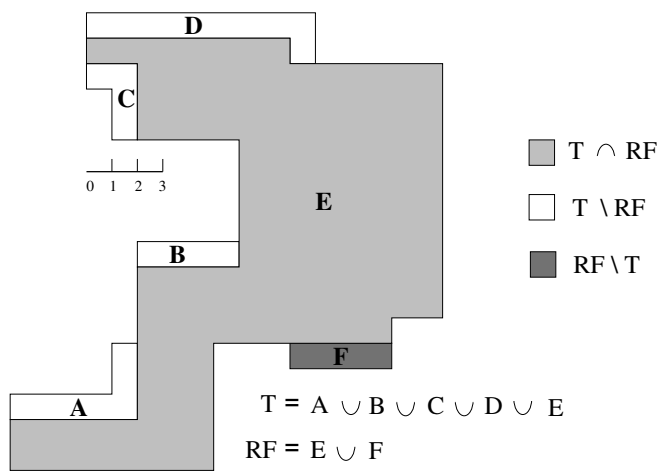

(a) Two compact sets $T$ and $R F$ with Hausdorff distance 1.

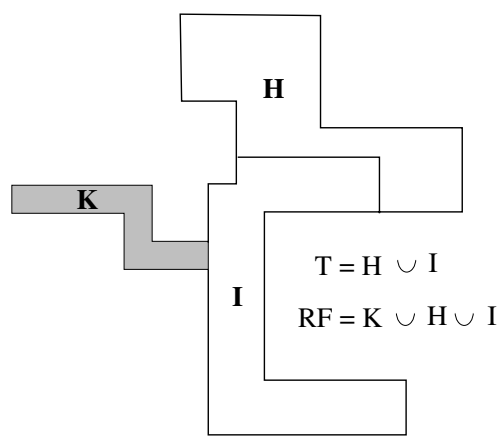

(b) Structural similarity of $T$ and $R F$

Fig. 4. Metric and structural comparison of receptive fields.

\section{Valuated Matroids}

In order to utilize local relinking operations for the adaption of a graph pyramid, the choice of the operations has to be determined by the goal of the adaption. We represent graph pyramids as bases of matroids and use a definition in [DW90, where $R$ denotes, for example, the set of reals or the set of integers.

Definition 3 (Valuation on a Matroid). A valuation on a matroid $\mathcal{M}=$ $\mathcal{M}(\mathcal{B})$ is a function $\omega: \mathcal{B} \rightarrow R$ which has the following exchange property. For $B, B^{\prime} \in \mathcal{B}$ and $b \in B \backslash B^{\prime}$ there exists $b^{\prime} \in B^{\prime} \backslash B$ such that

$-B \backslash\{b\} \cup\left\{b^{\prime}\right\} \in \mathcal{B}$,

$-B^{\prime} \backslash\left\{b^{\prime}\right\} \cup\{b\} \in \mathcal{B}$,

$-\omega(B)+\omega\left(B^{\prime}\right) \leq \omega\left(B \backslash\{b\} \cup\left\{b^{\prime}\right\}\right)+\omega\left(B^{\prime} \backslash\left\{b^{\prime}\right\} \cup\{b\}\right)$.

A matroid equipped with a valuation is called valuated matroid.

The following theorem [DW90] implies that valuations on matroids can be maximized by local modifications.

Theorem 3. Let $B \in \mathcal{B}$ and let $\omega$ be a valuation on the matroid $\mathcal{M}=\mathcal{M}(\mathcal{B})$. Then $\omega(B)$ is maximal, if $\omega\left(B_{m}\right) \leq \omega(B)$ for all local modifications $B_{m}$ of $B$.

In order to utilize Theorem 3 for the adaption of graph pyramids by local relinking operations, we have to find a valuation on the corresponding matroid, which is maximal if and only if the goal of the adaption is reached. Then we apply a local relinking operation whenever it increases the valuation. 


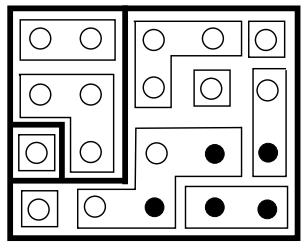

(a) $\mathcal{P}$
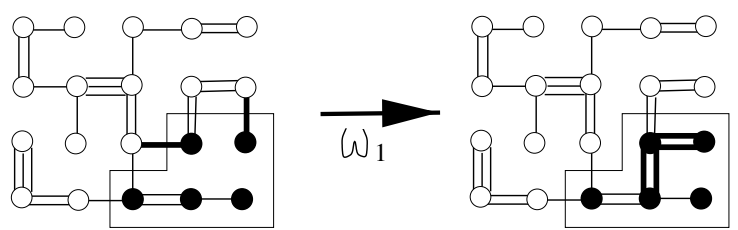

(b) Adaption guided by $\omega_{1}$

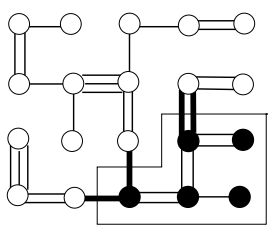

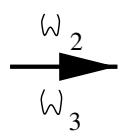

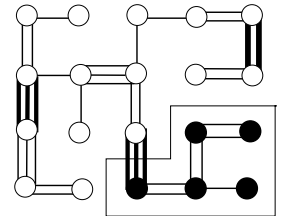

(c) Adaption guided by $\omega_{2}$ and $\omega_{3}$

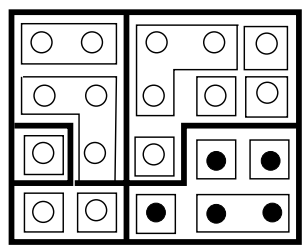

(d) $\mathcal{P}^{\prime}$

Fig. 5. Relinking towards a given receptive field. Modified edges are highlighted.

\section{Adaption of Graph Pyramids}

In this section we use valuated matroids to adapt a graph pyramid $\mathcal{P}$ towards having a receptive field equal to a given connected set $T$ of vertices from the base level of $\mathcal{P}$. If there is no receptive field equal to $T$, we may still ask: How well does $T$ fit into the pyramid $\mathcal{P}$ ? This question has a narrow metric and a wider structural aspect: If there exists a receptive field $R F$ in $\mathcal{P}$ with a small distance (Hausdorff-distance for example) to $T$, we say that $T$ fits well into $\mathcal{P}$. The wider structural aspect is the following: Can a good fit of $T$ into $\mathcal{P}$ be achieved by only a few (including zero) local relinking operations on $\mathcal{P}$ ? This case is illustrated in Fig. 4b, where splitting off the receptive field $K$ from $R F$ yields $T$.

In the following, we will apply local relinking operations to the graph pyramid $\mathcal{P}$, such that one of its receptive fields becomes equal to $T$. In Fig. 57 and $5 \mathrm{~d}$ the pyramid $\mathcal{P}$ and the adapted pyramid $\mathcal{P}^{\prime}$ are illustrated by their receptive fields. The set $T$ is given by the filled circles.

Since $T$ is contained in the receptive field of the apex of $\mathcal{P}$, there exists a smallest receptive field of $\mathcal{P}$ which covers $T$ completely. In particular, there exists a vertex $v_{T}^{\text {cov }}$ in $\mathcal{P}$ such that $T \subset R F\left(v_{T}^{\text {cov }}\right)$ and $T \not \subset R F(v)$ for all children $v$ of $v_{T}^{c o v}$. If $T=R F\left(v_{T}^{c o v}\right)$ no adaption of $\mathcal{P}$ is needed. Otherwise structural modifications are needed only in the subpyramid of $\mathcal{P}$, whose apex is $v_{T}^{\text {cov }}$.

As explained in Section [3, we may describe the adaption of $\mathcal{P}$ by local modifications on the corresponding matroid base $B$. The set $E_{0}$ of edges in the base 
level of $\mathcal{P}$ is partitioned by the edge sets $E^{0}(B)$ and $E^{1}(B)$, as defined in (2) and (3). The edge sets $E^{0}(B)$ and $E^{1}(B)$, in turn, are partitioned with respect to $T$ into three classes each. For $i \in\{0,1\}$ we set:

$$
\begin{aligned}
& -E_{1}^{i}(B):=\left\{e=(u, v) \in E^{i}(B) \mid\{u, v\} \subset T\right\}, \\
& -E_{2}^{i}(B):=\left\{e=(u, v) \in E^{i}(B) \mid\{u, v\} \cap T=\emptyset\right\}, \\
& -E_{3}^{i}(B):=E^{i}(B) \backslash\left(E_{1}^{i}(B) \cup E_{2}^{i}(B)\right) .
\end{aligned}
$$

Adapting $\mathcal{P}$ towards containing $T$ as a receptive field, we focus on the following edges in $E^{0}(B)$ :

Definition 4. An edge $e=(w, z) \in E^{0}(B)$ conflicts with $T$, if

$-e \in E_{3}^{0}(B)$ and

- one end point of e is contained in $R F\left(v_{T}^{\text {cov }}\right) \backslash T$.

Theorem 4. The graph pyramid $\mathcal{P}$ has no receptive field equal to $T \Leftrightarrow \mathcal{P}$ has edges conflicting with $T$.

\section{Proof of Theorem 4;}

$\Rightarrow$ : Assume that no receptive field of $\mathcal{P}$ equals $T$, i.e. $R F\left(v_{T}^{\text {cov }}\right) \supset T$ and $R F\left(v_{T}^{c o v}\right) \neq T$. The set of all edges from $E^{0}(B)$ with both end vertices in $R F\left(v_{T}^{c o v}\right)$ forms a spanning tree of $R F\left(v_{T}^{c o v}\right)$ and thus contains an edge $e=(w, z)$ with $w \in T$ and $z \in R F\left(v_{T}^{\text {cov }}\right) \backslash T$. The edge $e$ conflicts with $T$.

$\Leftarrow$ : Let $e=(w, z)$ be an edge conflicting with $T$. Without loss of generality we assume $z \in R F\left(v_{T}^{\text {cov }}\right) \backslash T$. It follows that $R F\left(v_{T}^{\text {cov }}\right) \neq T$. If there was a receptive field in $\mathcal{P}$ equal to $T, R F\left(v_{T}^{\text {cov }}\right)$ would equal $T$, a contradiction.

\subsection{Algorithm for the Adaption}

The adaption of $\mathcal{P}$ towards containing $T$ is done in three steps, all of which reduce the number of edges, which conflict with $T$ :

1. The number of edges in $E_{1}^{0}(B)$ is increased without affecting edges in $E_{2}^{0}(B)$.

2. The number of edges in $E_{2}^{0}(B)$ is increased without affecting edges in $E_{1}^{0}(B)$.

3 . The labels of the remaining edges conflicting with $T$ are raised.

In order to perform the first two steps, we define valuations $\omega_{1}$ and $\omega_{2}$. The matroid base $B$ is a subset of $E_{0} \times L$. An element $x$ of $B$ can be written as $x=\left(e_{x}, l\left(e_{x}\right)\right)$. For $e_{x} \notin E^{0}(B)$ let $C\left(B, e_{x}\right)$ denote the fundamental circuit of $E^{0}(B) \cup\left\{e_{x}\right\}$ and set

$$
l_{B}\left(e_{x}\right):=\max \left\{l(e) \mid e \in C\left(B, e_{x}\right), e \neq e_{x}\right\} .
$$

Let $e_{y} \in C\left(B, e_{x}\right)$ with $e_{y} \neq e_{x}, l\left(e_{y}\right)=l_{B}\left(e_{x}\right)$. In Kro95b it is shown that the graph pyramid defined by $B \backslash\left\{\left(e_{y}, l\left(e_{y}\right)\right)\right\} \cup\left\{\left(e_{x}, l_{B}\left(e_{x}\right)\right)\right\}$ equals the graph pyramid defined by $B$. For $i \in\{1,2\}$ we set $\omega_{i}(B):=\sum_{x \in B} v a_{i}(x)$ with

$$
\operatorname{val}_{i}(x):=\left\{\begin{array}{rll}
1 & : & e_{x} \in E_{i}^{1}(B), l\left(e_{x}\right)=l_{B}\left(e_{x}\right) \\
1 & : & e_{x} \in E_{1}^{0}(B) \cup E_{2}^{0}(B) \\
-1 & : & e_{x} \in E_{i}^{0}(B) \cup E_{i}^{1}(B), l\left(e_{x}\right) \neq l_{B}\left(e_{x}\right) \\
0 & : & \text { otherwise }
\end{array}\right.
$$


Consider the case $i=1$ first. The value 1 is given for labeled edges, which we want to insert between vertices of $T$. The same value is given for labeled edges that we do not want to change anymore. The valuation $\omega_{1}(B)$ is maximal only if the edges in $E_{1}^{0}(B)$ form a spanning tree of $T$. In the case $i=2$ the roles of $T$ and the complement of $T$ are reversed. Finally, the levels of the remaining edges conflicting with $T$ are raised to the highest even label $l_{a c c}$ an edge between vertices of $R F\left(v_{T}^{c o v}\right)$ can have. These local relinking operations are guided by the valuation $\omega_{3}(B):=\sum_{x \in B} \operatorname{val}_{3}(x)$ with

$$
\operatorname{val}_{3}(x):=\left\{\begin{array}{rll}
1 & : & e_{x} \in E_{1}^{0}(B) \cup E_{2}^{0}(B) \\
-1 & : & e_{x} \in E_{3}^{0}(B), l\left(e_{x}\right) \neq l_{a c c} \\
0 & : & \text { otherwise. }
\end{array}\right.
$$

Note that each local modification (guided by $\omega_{1}, \omega_{2}$ or $\omega_{3}$ ) reduces the number of edges conflicting with $T$ by exactly one and raises the valuation by exactly one. The effect on the receptive fields can each time be described as detaching a part of $R F\left(v_{T}^{c o v}\right)$. These parts are fully determined by the edges conflicting with $T$.

\subsection{Example}

Fig. 5b shows that there are exactly two local modifications which raise the valuation $\omega_{1}$. The total increase of $\omega_{1}$ thus amounts to 2 . Fig. 56 shows that $\omega_{2}$ and $\omega_{3}$ can be raised by 2 and 1 respectively. The comparison of Fig. 5 a and Fig. 5 d yields that none of the receptive fields completely contained in $T$ or completely contained in the complement of $T$ have been modified.

\section{Conclusion}

Valuations on matroids were shown to be capable of guiding the relinking of graph pyramids by local relinking operations. Furthermore, the local relinking operations may be performed in an iterated parallel way. We suggest the new method for tracking and motion analysis. In conjunction with dual graph contraction and contents adjustment it is also suggested for graph based object recognition.

\section{References}

[BHR81] P. J. Burt, T.-H. Hong, and Azriel Rosenfeld. Segmentation and estimation of image region properties through cooperative hierarchical computation. IEEE Transactions on Systems, Man, and Cybernetics, Vol. SMC11(No.12):pp.802-809, December 1981.

[Bru69] R.A. Brualdi. Comments on bases in dependence structures. Bull. Austral. Math. Soc., 21(3):161-167, 1969.

[DW90] W. M. Dress and Wenzel W. Valuated matroid: A new look to the greedy algorithm. Applied Mathmatics Letters, 3:33-35, 1990. 
[Kro95a] Walter G. Kropatsch. Building Irregular Pyramids by Dual Graph Contraction. IEE-Proc. Vision, Image and Signal Processing, 142(6):366 - 374, 1995.

[Kro95b] Walter G. Kropatsch. Equivalent Contraction Kernels and The Domain of Dual Irregular Pyramids. Technical Report PRIP-TR-42, Institute f. Automation 183/2, Dept. for Pattern Recognition and Image Processing, TU Wien, Austria, 1995.

[Nac95] Peter F.M. Nacken. Image segmentation by connectivity preserving relinking in hierarchical graph structures. Pattern Recognition, 28(6):907-920, June 1995.

[Ox192] J.G. Oxley. Matroid theory. Oxford University Press, New York, USA, 1992.

[Sch97] Andy Schürr. Handbook of Graph Grammars and Computing by Graph Transformations, chapter Programmed Graph Replacement Systems, pages 479-546. G. Rozenberg, 1997.

[Ser82] Jean Serra. Image and Mathematical Morphology, volume 1. Academic Press, London, G.B., 1982.

[TS92] K. Thulasiraman and M.N.S. Swamy. Graphs: Theory and Algorithms. J. Wiley \& Sons, New York, USA, 1992.

[WH96] Richard C. Wilson and Edwin R. Hancock. Hierarchical Discrete Relaxation. In Petra Perner, Patrick Wang, and Azriel Rosenfeld, editors, Proceedings SSPR'96, Advances in Structural and Syntactical Pattern Recognition, volume Vol. 1121 of Lecture Notes in Computer Science, pages 120-129, Leipzig, Germany, 1996. Springer, Berlin Heidelberg, New York. 